\title{
The Spitzer science operations system: how well are we really doing?
}

Suzanne R. Dodd

Suzanne R. Dodd, "The Spitzer science operations system: how well are we really doing?," Proc. SPIE 5487, Optical, Infrared, and Millimeter Space Telescopes, (12 October 2004); doi: 10.1117/12.551632

Event: SPIE Astronomical Telescopes + Instrumentation, 2004, Glasgow, United Kingdom 


\title{
The Spitzer science operations system: How well are we really doing?
}

\author{
Suzanne R. Dodd \\ California Institute of Technology \\ M/S 314-6, 1200 E. California Blvd, Pasadena, CA, 91125, USA
}

\begin{abstract}
The Spitzer Space Telescope was successfully launched on August $25^{\text {th }}$, 2003. After a 90 day In Orbit Checkout and Science Verification period, Spitzer began its five and one half year mission of science observations at wavelengths ranging from 3.6 to 160 microns. Early results from Spitzer show the observatory performing exceptionally well, meeting performance requirements in all areas. The California Institute of Technology is the home for the Spitzer Science Center (SSC). The SSC is responsible for selecting observing proposals, providing technical support to the science community, performing mission planning and science observation scheduling, instrument calibration and performance monitoring during operations, and production of archival quality data products. This paper will address the performance of the Spitzer science operations for the first nine months of the mission, covering science efficiency, science planning and scheduling metrics, data through-put and processing durations, system improvements, and science community interest. This work was performed at the California Institute of Technology under contract to the National Aeronautics and Space Administration.
\end{abstract}

Keywords: Spitzer Space Telescope, science operations, science planning, data processing, data archive

\section{INTRODUCTION}

\subsection{Mission overview}

The Spitzer Space Telescope is the fourth and final of NASA's great observatories. The observatory is designed to probe and explore the universe in the infrared at wavelengths ranging from 3.6 to 160 microns. The Spitzer observatory (Figure 1) consists of a Cryo-Telescope Assembly (CTA), spacecraft, and three science instruments: the Infrared Array Camera (IRAC), the Infrared Spectrograph (IRS), and the Multiband Imaging Photometer for Spitzer (MIPS). Each instrument consists of a cold assembly mounted in the cryostat and warm electronics mounted in the spacecraft bus. The CTA has an outer shell that radiates to cold space in the anti-Sun direction, and is shielded from the Sun by the solar panel assembly. The observatory is in an earth trailing, heliocentric orbit which eliminates the effect of heat from the Earth, allowing the unique cryogenic design to achieve an expected lifetime of $5+$ years.

Launched from Cape Canaveral, Florida, on August 25, 2003, the mission had planned for a 60 day InOrbit Checkout (IOC) period followed by a 30 day Science Verification (SV) phase. Spitzer employed a warm launch architecture and cooled to its final operating temperature of 5.6 degrees Kelvin over 45 days. IOC was dedicated to bringing the flight facility on-line safely and expeditiously, verifying the functionality of the instruments, telescope, and spacecraft. SV characterized the observatory's in-flight performance, commissioned the observing templates, and demonstrated that the ground software and operations processes were sufficient to commence routine operations. At the completion of SV, Spitzer transitioned into its normal operating mode, and has now completed six months of routine operations. 


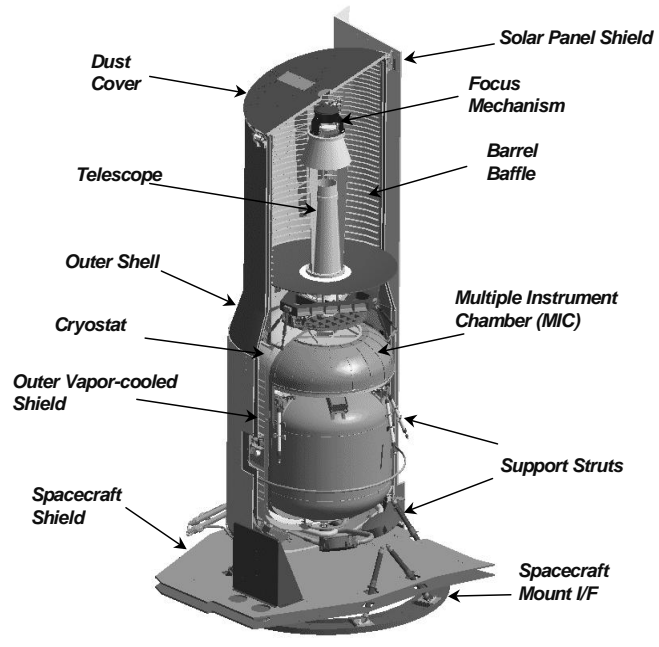

Fig. 1. Spitzer observatory

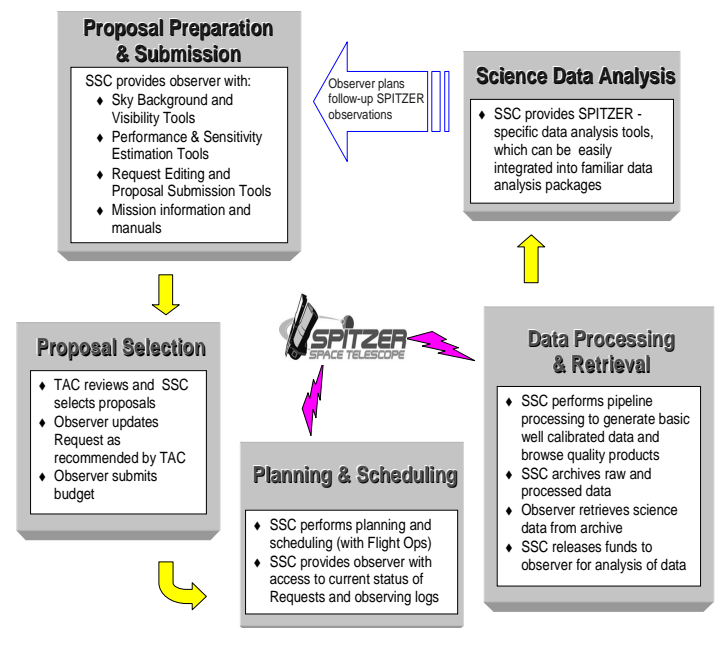

Fig. 2. SSC functional responsibilities

\subsection{Operations organization}

The Spitzer flight operations organization has two components - the Mission Operations System (MOS) at the Jet Propulsion Laboratory (JPL) and Lockheed Martin in Denver, where the engineering functions are performed, and the Spitzer Science Center (SSC) located at Caltech, where the science operations are performed. The SSC is responsible for evaluating and selecting observing proposals, providing technical support to the science community, performing mission planning and science observation scheduling, instrument calibration and instrument performance monitoring, data processing and production of archival quality data products, and funding science research (Figure 2). The uplink process starts at the SSC, with the generation of one week science schedules, which are then sent to JPL for final command generation, validation, and radiation to the observatory. Data are received from the observatory by the Deep Space Network (DSN) and transferred to JPL where level 1 data processing is done. The data is then sent to SSC for science data processing, science product generation, and archiving.

\subsection{Observatory performance}

The observatory performance against the functional requirements has been remarkable. All systems are performing very well. Predicted consumables usage for reaction control gas and thruster and latch valve cycles well exceeds five years. Helium gas, the limiting lifetime factor for Spitzer, is now estimated to last 5.2 years. Pointing performance of the observatory is excellent. The Sun sensors, star trackers, gyros, and pointing control reference sensor are all calibrated and meet or exceed requirements. The absolute pointing accuracy, blind pointing, offset pointing, and moving object tracking all meet or exceed requirements. This exceptional spacecraft performance allows the quality of the scientific data to far exceed that of any previous infrared mission. 


\section{SCIENCE OPERATIONS}

\subsection{IOC/SV operations}

The Spitzer IOC and SV phases were planned to last for 90 days. Ground operations were event-driven and expected to be intense compared to normal operations. Many on-board activities were dependent on the results of precursor activities. While some activities could be carried out early with a warm telescope, most of the instrument activities required a much cooler telescope and were scheduled later in the IOC/SV period. Rescheduling and resequencing had to be flexible and rapid to maximize the productive use of flight time.

The Spitzer flight team completed IOC in 62.8 days and SV in 35.6 days for a total of 98.4 days. The longer duration was due primarily to the three safing/standby events experienced by the spacecraft. One of these events was the "solar storm of the century" on October 28, 2003. The event required the observatory to be idled for two and a half days. Another two days was spent carrying out the solar storm recovery procedures for the science instruments. The completion of the IOC and SV phases within a week of the planned end is a remarkable achievement, and is due to the hard work and dedication of the whole Spitzer project, including the SSC, the MOS, and the instrument principal investigator teams.

\subsection{Science efficiency}

Science efficiency is the measure of time spent actually executing science observations. As defined for Spitzer, this includes the time spent slewing to an observation and the time used for science instrument (SI) calibrations. It does not include spacecraft $(\mathrm{S} / \mathrm{C})$ calibration time or data downlinks $(\mathrm{D} / \mathrm{L})$. As of the writing of this paper, Spitzer has executed 25 weeks of normal operations. The science efficiency has risen steadily over this time period (Figure 3). Our pre-launch proof-of-concept (POC) exercise estimate for science efficiency was 85.6\% (Figure $4 \mathrm{~b}$ ) as compared to the on-orbit science efficiency of $83 \%$ (Figure 4a). The POC was based on a scheduling order of 10 days of IRAC, 7 days of MIPS, and 5 days of IRS. The nominal instrument ordering has turned out to be 9 days of IRAC, 8 days of MIPS, and 6 days of IRS. The Spitzer project goal for science efficiency is $90 \%$, and the project is close to accomplishing that.

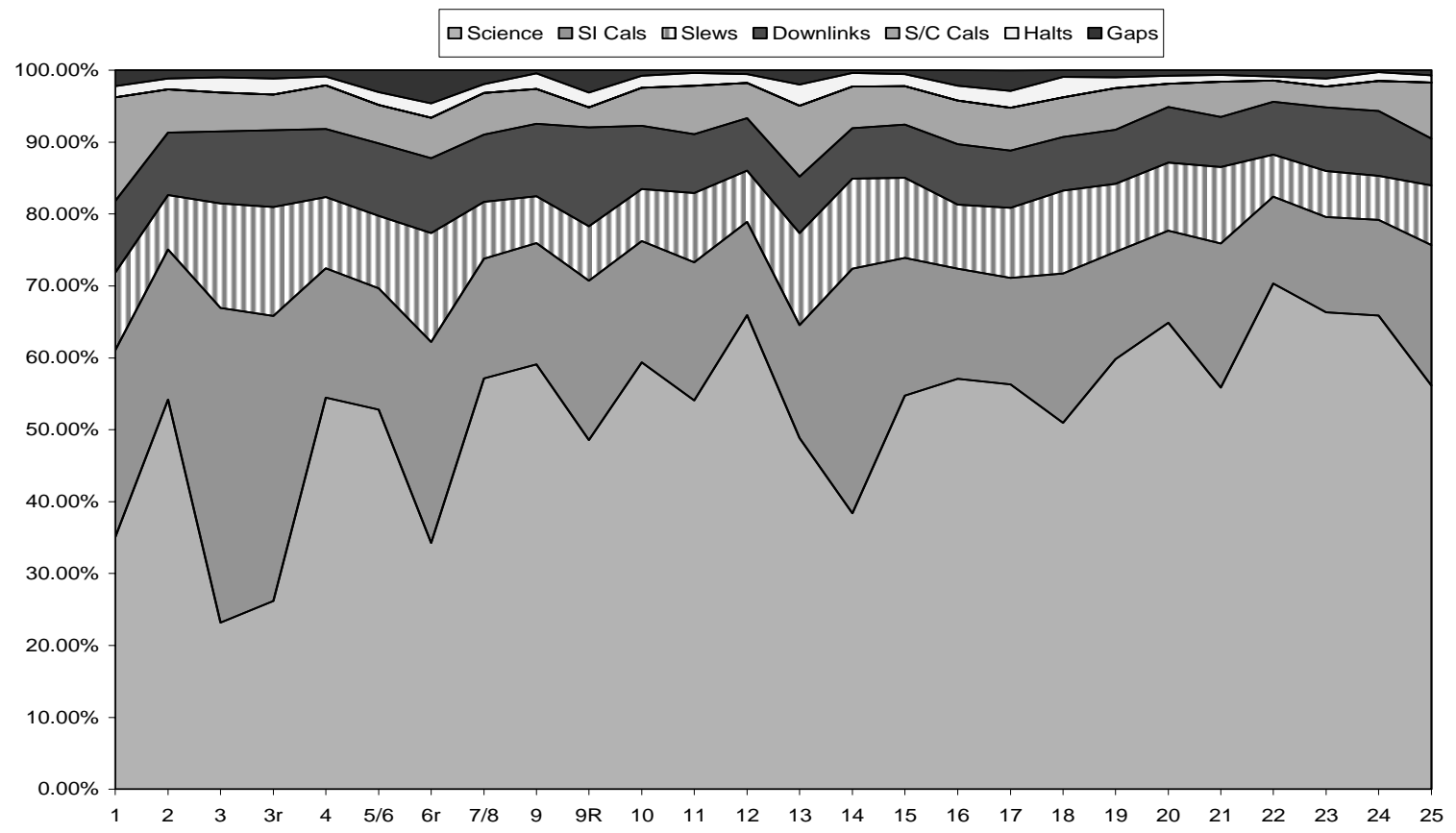

Fig. 3 Science efficiency 


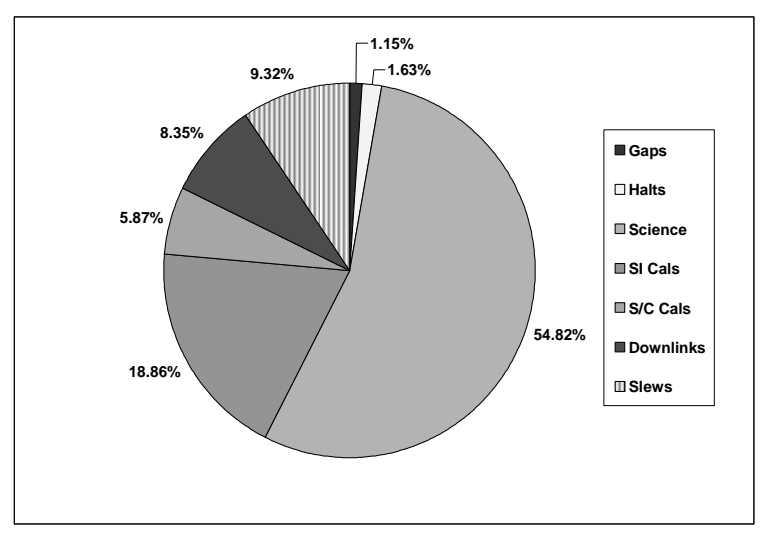

Fig. 4a On-orbit efficiency

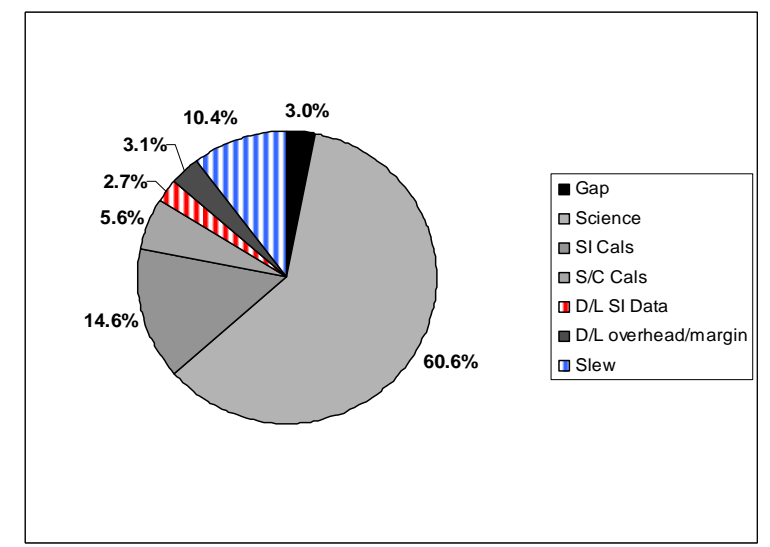

Fig. 4b Proof-of-concept efficiency

Several changes have been made to improve efficiency. The duration and frequency of boresight calibrations has been reduced, saving five hours of spacecraft calibration time per week. This change was implemented starting in week 20. A change to the pointing control system maximum torque configuration has been implemented, reducing the slewing time for short slews and saving on average 3 hours of time per week. The duration of the downlink passes has been tuned to match the downlink volume duration during the over night pass. The day time pass is 50 minutes, with the time not taken by the data downlink used for engineering commanding if needed. Early in the mission both daily passes were full one hour passes. Finally, the scheduling algorithm in the ground software has been tuned to do a better job of optimizing slewing.

Areas of efficiency increase continue to be identified. Grouping together calibration observations to minimize slewing and, where possible, selecting targets in the same part of the sky as science observations, will free up more time for science observations. Reducing the frequency of gyro calibrations is also being considered to improve observing efficiency. These types of improvements are part of a continuous, deliberate effort to maximum the amount of science obtained from Spitzer.

\subsection{The uplink process}

The transition from the IOC/SV rapid, event-driven uplink process to the standard 40 hour a week normal operations process was a difficult one. The IOC/SV process was designed to allow for short lead times, while the normal process involves almost six weeks of development time (Figure 5). It took 12 weeks of normal operations before we fully achieved the standard operations uplink timeline. Figure 6 is a graphic of the duration of development time for each weekly sequence. The sequence development time is divided into three parts: sequence packaging, sequence generation pass 1 , and sequence generation pass 2 . Since week 16, the uplink process milestones have consistently been met, and in some cases we have even had sequences developed ahead of schedule (for example week 19). 


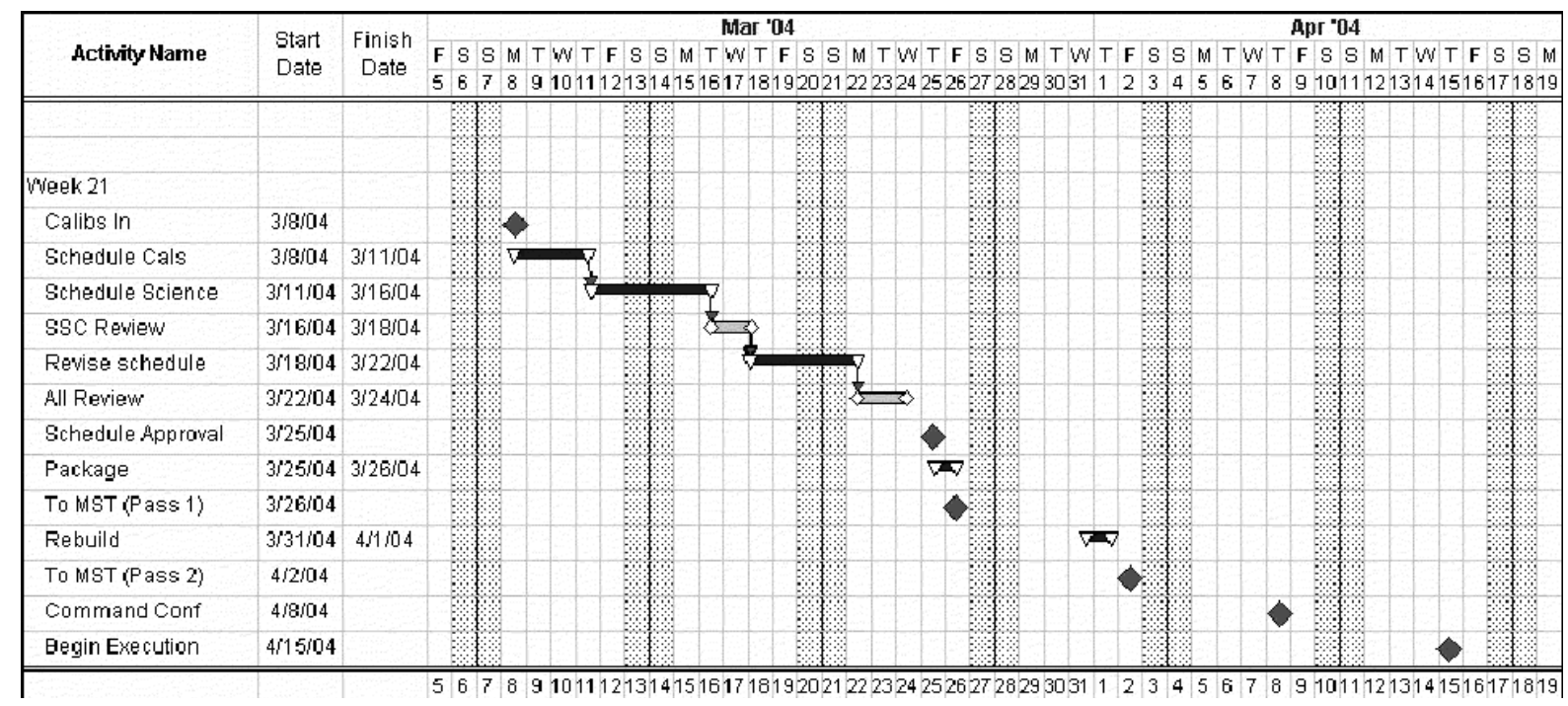

Fig. 5 Uplink process timeline

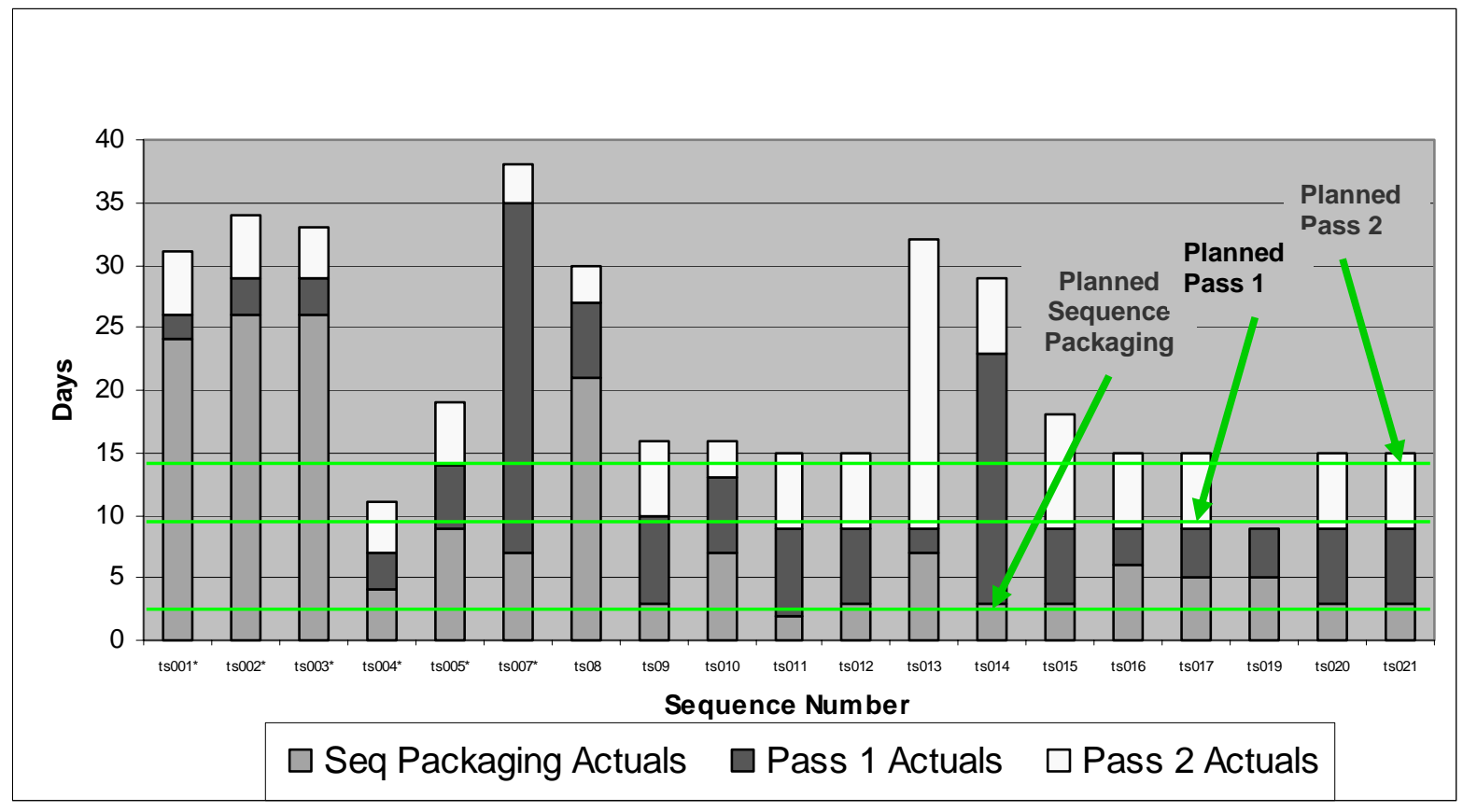

Fig. 6 Sequence development metrics

There are still several areas where the scheduling process could be improved. Currently the SSC schedulers must handle bright object observations manually, following prescribed rules. This is labor intensive and adversely affects the efficiency of the process. Database infrastructure and software updates are planned to flag observations which need this type of special handling. Also, several observation programs are highly constrained as to when and how they can occur. These observations drive the scheduling process with other less constrained observations scheduled around them. The interaction of constrained programs tends to increase slewing and thus reduces science efficiency. Constrained programs also substantially complicate the recovery from any on-board anomaly. 


\subsection{Data Processing}

There are several downlink and data processing requirements for Spitzer, all of which are being met. The data transfer requirement of less than 12 hours from the spacecraft downlink to receipt at SSC is routinely met, with the median time being less than 90 minutes. The percentage of missing science data over the first six months of normal operation is $0.04 \%$, well below the requirement of $2.0 \%$. Analysis of the data quality has found only four observations requiring repeat observations out of a total of 3440 executed.

The SSC data pipelines used to process the data have all been commissioned and are being used to process data for archival. The pipeline processing through-put times are: 25 times realtime for IRS (i.e. we can reprocess data 25 times faster than the speed at which it is obtained), 5 times realtime for IRAC, and 3 times realtime for MIPS. The goal for reprocessing speed is 5 times realtime, and we are actively working on ways to improve the current MIPS data processing time. Currently, the observer gets the data three to four weeks after the observation is made. During that time the data quality is assessed, the data are reprocessed with updated calibration files, archived, certified and released. The goal for this data delivery process is two weeks or faster.

\section{COMMUNITY INTEREST}

\subsection{Metrics}

Science community interest in Spitzer is very high. Tickets to the observer support team helpdesk reached a peak of 773 in February, corresponding to the close of the general observer cycle 1 proposal call (Figure 7). Similarly, hits to the Spitzer public website, www.spitzer.caltech.edu, reached high points in December and January with the release of the first Spitzer images, and peaked in March at over 10 million with the announcement of Spitzer's support of the planetoid Sedna discovery (Figure 8). Both SSC standing review boards, the Science Users Panel and the Oversight Committee, met in April and each expressed the science community's excitement over the quality of the data and the success of the mission to date.

\begin{tabular}{|c|c|}
\hline MONTH & TICKET ACTIVITY \\
\hline August 2003 & 96 \\
\hline September 2003 & 80 \\
\hline October 2003 & 90 \\
\hline November 2003 & 254 \\
\hline December 2003 & 390 \\
\hline January 2004 & 440 \\
\hline February 2004 & 773 \\
\hline March 2004 & 171 \\
\hline April 2004 & 268 \\
\hline
\end{tabular}

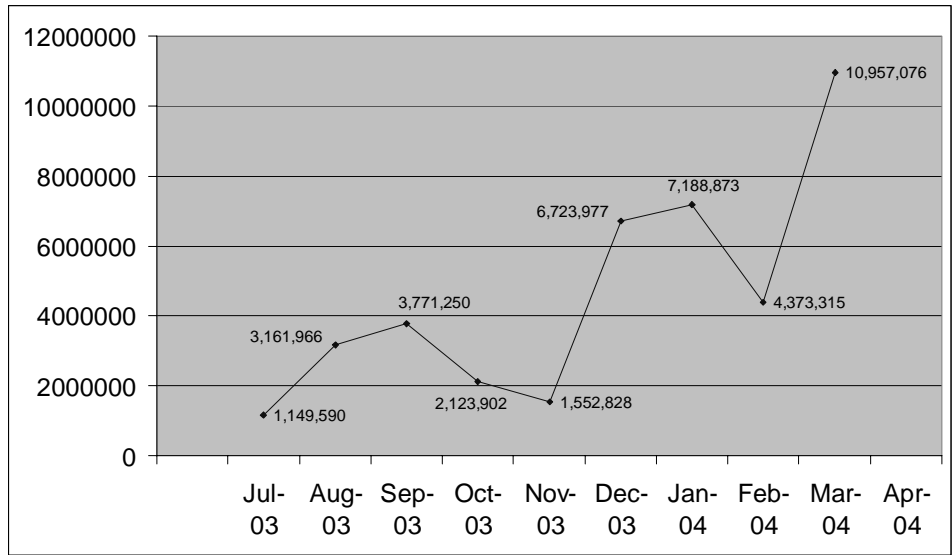

Fig. 7 Helpdesk tickets

Fig. 8 Public website hits 


\subsection{Observation summary}

The first seven months of normal operations are being spent executing observing programs of the guaranteed time observers (GTOs) and legacy science teams. There are six legacy science teams who each have 350-850 hours of observing time to execute large, systematic data collection programs in both the galactic and extragalactic disciplines. Those data are non-proprietary, and go into the public archive as soon as campaign processing is finished. These data will provide the basis for follow-up observations, as well as leaving a scientific legacy for Spitzer. The final category of observing time is the Director's discretionary time (DDT). This time is allocated by the SSC director for special observations of time-critical, high valued science. Figure 9 is a chart showing the breakdown in observations taken through the month of April.

\begin{tabular}{|c|c|c|c|c|c|c|}
\hline Program Type & $\begin{array}{c}\text { Total } \\
\text { Planned } \\
\text { Requests }\end{array}$ & $\begin{array}{c}\text { Total } \\
\text { Planned } \\
\text { Hours }\end{array}$ & $\begin{array}{c}\text { Requests } \\
\text { Executed to } \\
\text { Date }\end{array}$ & $\begin{array}{c}\% \text { of Total } \\
\text { Planned } \\
\text { Requests }\end{array}$ & $\begin{array}{c}\text { Request } \\
\text { Hours to Date }\end{array}$ & $\begin{array}{c}\% \text { of Total } \\
\text { Planned } \\
\text { Hours }\end{array}$ \\
\hline GTO & 5924 & 3361 & 1992 & $33.60 \%$ & 1188 & $35.30 \%$ \\
\hline Legacy & 3518 & 3210 & 547 & $15.50 \%$ & 682 & $21.20 \%$ \\
\hline \hline DDT & 355 & 223 & 191 & $57.70 \%$ & 164 & $73.50 \%$ \\
\hline \hline Totals & 9797 & 6794 & 2730 & $28 \%$ & 2034 & $30.00 \%$ \\
\hline
\end{tabular}

Fig 9. Observation summary

\subsection{General observer cycle 1}

The general observer (GO) cycle-1 proposal call resulted in 592 proposals being received. 574 proposals were for general observing time and 18 were for archival research. Fully $85 \%$ of the principal investigators have no connection to Spitzer and $26 \%$ are led by a non-US scientist, demonstrating that Spitzer time is sought after in the broader world-wide community of infrared astronomers. The proposals added up to 13,746 hours of observing time, where 3700 hours is available, giving an over subscription rate of 3.7. 36 proposals are classified as medium size (50-200 hours of observing time), and 538 are small proposals (less than 50 hours of observing time). $44.5 \%$ of the proposals propose studying extragalactic objects, $48.9 \%$ propose doing galactic science, and $6.6 \%$ propose looking at solar system targets. As of this writing, the time allocation committee (TAC) is meeting to decide which of these proposals will be selected for the GO-1 cycle which runs from late June 2004 to late June 2005. It's worth noting that all the software, people, and processes involved in the proposal creation, ingestion, documentation, and review system worked extremely well. A few minor problems have been reviewed, with modifications planned for the GO cycle 2.

\section{CONCLUSIONS AND ACKNOWLEDGEMENTS}

Over twenty years of planning has gone into the Spitzer Space Telescope. The successful launch and operations of Spitzer is a dream come true to many in the infrared astronomical community. The data being returned are "lifting the cosmic veil" of the universe. The first three months of operations during IOC/SV were hectic, but since then the science operations has settled into a routine mode. As good as Spitzer operations are, the SSC will continue to implement ways to improve science efficiency on board the observatory, and to improve the scientific quality of the data. With a lifetime expectancy of greater than five years, Spitzer will indeed be a truly great observatory for NASA. 
The success of the Spitzer science operations system is due to the efforts of the outstanding staff of the Spitzer Science Center in designing, developing, implementing, testing and operating the system. Many SSC staff members have worked on the project for several years, and are just now seeing the fruits of their labor. It is a privilege to manage such a talented and dedicated organization. This work was performed at the California Institute of Technology under contract to the National Aeronautics and Space Administration.

\section{REFERENCES}

1. S.H. Linick, J.W. Miles, C.A. Boyles, J.B. Gilbert, Spitzer Space Telescope In-Orbit Checkout and Science Verification Operations, SpaceOps 2004 Conference, Montreal, Canada, May 17-21, 2004. 